\title{
PLAN DE MANEJO DE LOS BOSQUES EN LOS SISTEMAS AGROFORESTALES CAFETALEROS DE JIPIJAPA, MANABÍ, ECUADOR.
}

PLAN OF FOREST MANAGEMENT IN AGROFORESTRY COFFEE, JIPIJAPA, MANABÍ, ECUADOR.

MSc. Blanca S. Indacochea Ganchozo ${ }^{1}$; Dr.C. Maurilio R. García López²; Dr.C Rogelio Sotolongo Sospedra ${ }^{2}$; Econ. Blanca Viviana Álvarez Indacochea'; Ing. Johann Parrales Villacreses ${ }^{1}$; Ing. Otto Quimís Garcés ${ }^{1}$.

${ }^{1}$ Universidad Estatal del Sur de Manabí. Av. Los Ángeles- vía a Noboa. JipijapaManabí. blancaindacochea@hotmail.com

2 Universidad de Pinar del Río, Martí No. 270, esq. 27 de noviembre, Pinar del Río, Cuba.

\section{RESUMEN}

Los agroecosistemas cafetaleros han recibido especial atención por su aparente capacidad para proteger la biodiversidad, preservar hábitats y recursos, especialmente en áreas con pequeños bosques. La intensificación del manejo de los cafetales, sin embargo, provoca pérdida de diversidad. El café fue cultivado tradicionalmente bajo una diversa y densa cobertura, pero los métodos modernos de cultivo se caracterizan por su reducción y los pobladores tienen una baja percepción sobre los bienes y servicios ambientales de los bosques secundarios en los sistemas agroforestales cafetaleros.

Este programa se propone servir de modelo en el cantón para la integración de las acciones de investigación científica y adopción de tecnologías para conservar la biodiversidad y promover el desarrollo comunitario sostenible. Considera acciones estratégicas para acumular información, desarrollar metodologías de trabajo y 
desarrollar técnicas para la rehabilitación de hábitat, la promoción de la agricultura sostenible, y actividades de educación ambiental comunitaria.

Por lo que el objetivo general del trabajo es proponer una estrategia para la gestión sostenible de los bosques ocupados por fincas agroforestales en el cantón Jipijapa.

Palabras clave: agroforestales,agroecosistemas cafetaleros. Plan de manejo, sistemas,

\section{ABSTRACT}

PLAN OF FOREST MANAGEMENT IN AGROFORESTRY COFFEE, JIPIJAPA, MANABÍ, ECUADOR.

The Coffee agroecosystems have received particular attention for its apparent ability to protect biodiversity, preserve habitats and resources, especially in areas with small forests. The intensification of coffee management, however, causes loss of diversity. Coffee was traditionally grown under a diverse and dense cover, but modern farming methods are characterized by reduction and villagers have a low perception of environmental goods and services of secondary forests in coffee agroforestry systems.

This program is intended as a model in the canton for integrating scientific research activities and adoption of technologies to conserve biodiversity and promote sustainable community development. Consider strategic actions to gather information, develop methodologies and develop techniques for habitat rehabilitation, promotion of sustainable agriculture, environmental education and community activities.

So the aim of this paper is to propose a strategy for the sustainable management of forests occupied by agroforestry farms in the canton Jipijapa.

Key words:agroforestry, coffee agroecosystems, Management plan 


\section{INTRODUCCCIÓN}

El bosque neotropical posee una capacidad extraordinaria para recuperar su biodiversidad vegetal y sus funciones, aun cuando un sitio haya sido completamente deforestado. La sucesión que tiene lugar en el bosque luego de que este ha sido despojado de la cobertura boscosa depende de la actividad realizada en el sitio previo a su abandono, la intensidad con que se llevó a cabo dicha actividad, la presencia o no de vegetación remanente y las características físicas interactúan de forma compleja para producir en un momento y espacio dado una comunidad particular de plantas (Guariguata y Kattan, 2002).

La conservación y utilización racional de los recursos forestales constituye un importante desafío de carácter global, por cuanto conseguir un adecuado equilibrio entre la utilización y conservación de estos recursos representa un aspecto crucial para el desarrollo (Quédraogo, 1997).

Los bosques degradados pueden cumplir una variedad de funciones sociales, productivas y de protección que podrían ser beneficiosas tanto para la seguridad alimentaria de la población como para el medio ambiente (Scherr, 2003). La degradación forestal se ha definido como la reducción de la capacidad de un bosque para producir bienes y servicios (OIMT, 2002).

El desarrollo de criterios e indicadores para la verificación de la sostenibilidad de la gestión Forestal ha surgido debido a dos causas:

- La necesidad de los gobiernos exponer el progreso hacia una conservación de los recursos forestales. 
- Demanda de mercado en muchos casos estimuladas por grupos ambientalistas y otro grupos sociales, de productos originarios de sistemas forestales bien gestionados.

La idea de la gestión sostenible de las masas forestales es considerada como un hecho positivo, ya que las compañías lo perciben como un modo de contentar al cliente y muchos forestales como un reto interesante para optimizar la gestión de cara al siglo XXI (Amezaga yOnaindia, 1997).

Hay una creciente preocupación por la pérdida de especies y hábitats, la erosión de la diversidad bajo un impacto humano cada vez mayor y la modificación de los procesos que la modelan. Al mismo tiempo, existe un desafío marcado por la complejidad de los ecosistemas y por la ignorancia de los mecanismos que sustentan la diversidad biológica (García, 2006).

En Ecuador los esfuerzos han sido dirigidos a la conservación de especies de interés agropecuario y aún son muy limitados los estudios ecológicos-genéticos en especies forestales (Grijalva et al., 2012, Tapia et al., 2008). Simultáneamente, se promulgan planes de reforestación para especies prioritarias (Bodero A, 2005.), sin que existan estudios propios del recurso genético y las posibles áreas de recolección o áreas semilleras, por lo que la transferencia y certificación de material superior proveniente de programas de mejora forestal aún es una quimera.

A excepción de evaluaciones a niveles muy locales de especies como Tabebuiachrysantha, Swieteniamacrophylla y Cordiaalliodora como elementos de los bosques secos y tropicales (Holl et al, 2000), en ensayos a gran escala en Ochromapiramidale (Grijalva et al., 2012,) y del Ministerio de Agricultura sobre la disponibilidad de los recursos genéticos (MAGAP,2003), existen muy pocos estudios 
detallados sobre la ecología, manejo de la semilla y de diversidad de bosques donde se encuentran las especies prioritarias.

El primer estudio incorporando elementos de la ecología y la gestión del recurso Cordiaalliodora en Jipijapa fue realizado por Indacochea-Ganchozo 2010a, b, 2011, el cual representa un acercamiento a la problemática de los impactos de la actividad agroforestal sobre los remantes de bosques y donde Cordiaalliodora fue determinada como especie clave y a la identificación entonces de una nueva problemática relacionada con la conservación y fomento de plantaciones de dicha especie clave.

\section{METODOLOGÍA}

Para caracterizar la percepción de los agricultores de las parroquias sobre los bienes y servicios del bosque y específicamente sobre el uso de la especie Cordiaalliodora se realizaron entrevistas individuales. Se tuvo en cuenta para el caso de los bienes y servicios el listado presentado por Arcia y Garibaldi (2005) para los fragmentos de bosques en la reserva forestal El Montuoso.

Se indagó también sobre la percepción de los pobladores acerca del impacto del manejo de estos sistemas agroforestales sobre el estado de conservación de los bosques.

Algunos aspectos importantes considerados en la aplicación de la entrevista es que en su mayoría los agricultores no saben leer ni escribir; la densidad de la población es baja por la emigración a la ciudad y las viviendas están dispersas.

\section{$\underline{\text { Muestreo }}$}

Se entrevistaron 105 agricultores, repartidos proporcionalmente, 15 de cada una de las siete parroquias rurales: América, El Anegado, Pedro Pablo Gómez, Julcuy, La Unión, Membrillal y Puerto Cayo.

\section{RESULTADOS}


Percepción sobre los impactos del manejo

En general es muy baja la percepción de los pobladores sobre las consecuencias del manejo que realizan en sus predios, el componente forestal de los agroecosistemas cafetaleros no constituye un aspecto primordial y solo es valorado desde el punto de vista material (la comercialización de la madera).

Bienes y servicios reconocidos por los entrevistados

Una forma de valorar económicamente los servicios ambientales es mediante la determinación del valor del uso que se le da al bien o servicio, el cual puede ser directo o indirecto.

La totalidad de los entrevistados reconocen como bienes directos del bosque los siguientes:

- Madera aserrada

- Leña y carbón

- Plantas medicinales

- Madera para varas, horcones, soleras, postes

- Plantas comestibles

Los servicios indirectos más reconocidos fueron:

- Energía

- Regulación hídrica

- Control de la erosión

En cuanto a la percepción de los agricultores sobre los usos y conocimiento de la $C$. alliodorase obtuvo que:

La totalidad conoce la especie

Reconoce como usos generales: 
- Ebanistería

- Construcción

Solo el $3 \%$ le atribuye usos medicinales y el $4 \%$ como combustible.

Como usos más específicos reconocen:

- Construcción de interiores

- Cubiertas de buques

- Carrocería de camiones

- Remos

- Durmientes

- Botes

- Melífera

- Ornato

- Chapas decorativas

Valoración sobre los servicios ambientales del bosque

Los pobladores identifican como servicios ambientales los siguientes:

- Conservación de la biodiversidad (70\%)

- Preservación del recurso agua (100\%)

- Control de la erosión edáfica (86\%)

- Refugio y fuente de alimentos para la fauna silvestre (51\%)

- Plantas medicinales (96\%)

Menos identificados son:

Los aportes del bosque en la polinización, control biológico, recursos genéticos, o recursos bioquímicos pasando totalmente inadvertidos.

DISCUSIÓN 
Los agricultores perciben en gran magnitud su dependencia del recurso madera, pero no toman acciones específicas para conservar el recurso y garantizar su permanencia a largo plazo. Tampoco toman acción alguna para la conservación de los suelos en las áreas dedicadas a la agricultura, aunque sí reconocen la existencia de algunas técnicas para ello y de la baja fertilidad natural de los suelos.

De acuerdo a los resultados obtenidos por las entrevistas y la encuesta aplicadas a los pobladores de las parroquias objeto de estudio no existe una percepción clara acerca de las consecuencias del manejo y su impacto sobre los remanentes de bosques en los sistemas agroforestales. Las necesidades materiales derivadas de la pobreza imponen un uso de los recursos que atenta contra su integridad.

El problema planteado es crítico en la mayor parte de las áreas rurales de Mesoamérica y países en desarrollo en general, donde aún se conserva y sobrevive la mayor parte de la diversidad biológica global y donde la pobreza tiende a ser el problema medular. Por ello, la armonización del desarrollo económico con la conservación de la biodiversidad se ha convertido en uno de los elementos prioritarios para alcanzar el anhelado "desarrollo sostenible" (FAOa, 2005; FAOb, 2005).

Al respecto, un estudio de la CEPAL señala que aún cuando la reducción de la pobreza rural no siempre disminuirá, el manejo adecuado de los recursos naturales sí podría contribuir a frenar la destrucción del medio natural, en donde existe presión de la población local sobre los frágiles y/o deteriorados recursos (Espinoza et al., 1999). Por ello, las opciones para conservar la diversidad biológica deben considerar la inclusión de incentivos económicos para aumentar los beneficios locales de la conservación y el uso sostenible de los recursos (McNeely, 1988).

En cuanto los servicios ambientales del bosque, como concepto, no son claramente identificados por la población, tal vez por lo reciente de la incorporación de este 
vocablo en la gestión ambiental. Este desconocimiento agudiza la problemática de la conservación pues no hay una percepción del riesgo que supone la destrucción total de estos bosques.

Uno de los aspectos que se destaca de este estudio es la prioridad y el conocimiento que tienen los pobladores sobre la especie $C$. alliodora, el estudio florístico la identifica como la de mayor índice de valor de importancia ecológica por lo que se deduce que el manejo la ha favorecido.

Desde el punto de vista de la conservación de la diversidad florística este puede ser un aspecto negativo pues se está favoreciendo una especie en detrimentos de las otras. Esta situación tiene una explicación económica, C.alliodoratiene una excelente madera, y está bien valorada en el mercado, por otra parte es una especie de crecimiento relativamente rápido y su manejo no resulta complejo en poblaciones puras.

Ello sugiere su potencial para ser incorporadas a un programa de manejo forestal para la recuperación de estos bosques. Estos datos apoyan lo argumentado (Laurance y Bierregaard, 1997) de que la sobrevivencia de muchas especies de la biota tropical actual dependerá de la habilidad de las especies de persistir en hábitats altamente modificados y de nuestra capacidad de manejar y administrar tales paisajes degradados.

No obstante en varias parroquias $C$. alliodora presenta irregularidades debido a la ausencia de suficientes individuos juveniles. Es posible que la falta de control de un diámetro mínimo de corta pudiera estar favoreciendo la extracción de los individuos juveniles que no están alcanzando su etapa reproductiva, por lo tanto no se observó suficiente regeneración de propágulos en la mayoría de las parroquias. 
De acuerdo al análisis de los resultados se confirma la hipótesis planteada sobre la baja percepción que tiene la comunidad sobre el significado e importancia de los bosque secundarios en los agroecosistemas cafetaleros de Jipijapa, situación que pone en peligro la conservación de estos remanentes de bosque y su aporte a la estabilidad medio ambiental de la región.

Propuesta de lineamientos estratégicos para un plan de acción para la conservación, restauración y manejo de los bosques en sistemas agroforestales cafetaleros del Cantón Jipijapa

Aspectos considerados en la propuesta:

La propuesta se basa en los lineamientos generales enunciados por García (2006) para el diseño de programas de gestión y estrategias de conservación de especies forestales, el cual constituyen un documento importante para todas las instituciones involucradas en la conservación de los recursos genéticos y forestales.

La propuesta ha sido formulada considerando la información recopilada durante la investigación y el trabajo e intercambio continuo con los líderes de las parroquias llevados a cabo con estudiantes de la carrera de ingeniería Forestal de la Universidad Estatal del Sur de Manabí.

Para la elaboración de la propuesta se ha considerado lo siguiente:

- La información sobre la diversidad, riqueza y estructura de los bosques de las parroquias del cantón recabada durante esta investigación.

- La identificación de impactos de la extracción tradicional de productos del bosque desarrollados durante esta investigación.

- El estudio sobre la percepción de los bienes del bosque por los agricultores de las parroquias del cantón. 
- Los resultados de reuniones, entrevistas y talleres de consulta efectuados con los distintos agricultores de las parroquias.

- Las recomendaciones de expertos relativas a criterios básicos para la conservación y utilización racional de los recursos forestales

Las directrices o elementos principales sugeridos, a considerar en la elaboración del plan son los siguientes:

- Aplicación de medidas de protección y manejo de la biodiversidad existente.

- Promoción de la recuperación de bosques en sistemas agroforestales cafetaleros del cantón Jipijapa.

- Promoción de buenas prácticas de manejo de recursos naturales en las actividades productivas.

La estrategia propuesta sugiere incluir los siguientes aspectos:

- Planificación comunitaria participativa.

- Capacitación técnica a los agricultores de las parroquias del cantón Jipijapa

- Combinación de actividades de agricultura y manejo forestal

- Programas de extensión en las parroquias del cantón Jipijapa

- Evaluación participativa

Los principales programas identificados son:

- Programa de rehabilitación y manejo de ecosistemas

- Programa de producción sostenible y extensión

- Programa de investigación y monitoreo

- Programa de educación ambiental y divulgación

De éstos programas se derivan 13 líneas de acción, que son:

$\checkmark$ Manejo de bordes en las fincas de cafetales. 
$\checkmark$ Recuperación de zonas desforestada (aislamiento de áreas con intervención humana grave y enriquecimiento de especies).

$\checkmark$ Plantaciones con especies nativas aptas según objetivos.

$\checkmark$ Colecta y manejo de semillas forestales y frutales.

$\checkmark$ Cultivo y manejo de especies nativas.

$\checkmark$ Producción de plantas nativas para reforestación en viveros comunitarios.

$\checkmark$ Estudios de regeneración natural de especies claves: Cordiaalliodora,Swieteniamacrophylla,Chlorophoratinctoria, Cedrelaodorata (regeneración, crecimiento, sobrevivencia, mortalidad, etc.).

$\checkmark$ Estudios fenológicos de especies forestales nativas

$\checkmark$ Cambios en la estructura del bosque debido a los disturbios naturales y antrópicos.

$\checkmark$ Fortalecimiento de grupos locales, organizaciones campesinas en temas ambientales. Importancia de la organización.

$\checkmark$ Organización de grupos estudiantiles locales en educación ambiental.

$\checkmark$ Participación en Ferias regionales de las parroquias: Pedro Pablo Gómez, Membrillal, Julcuy, Puerto Cayo, El Anegado, La Unión y América

$\checkmark$ Participación en Encuentros de agricultores, estudiantiles y comunidad

Como resultado de estos esfuerzos se obtuvo lo siguiente:

La conformación de un Comité integrado por miembros de las Juntas Parroquiales, Ministerio del Ambiente e investigadores académicos que supervisa la ejecución de un programa piloto de investigación participativa.

"Programa piloto para promover la conservación de la diversidad biológica y el desarrollo sostenible de las parroquias 
Este Programa se propone servir de modelo en el Cantón para la integración de las acciones de investigación científica y adopción de tecnologías para mitigar la pobreza, conservar la biodiversidad y promover el desarrollo comunitario sostenible. Considera acciones estratégicas para acumular información, desarrollar metodologías de trabajo y desarrollar técnicas para la rehabilitación de hábitat, la promoción de la agricultura sostenible, y actividades de educación ambiental comunitaria.

Sustentabilidad a largo plazo

La sustentabilidad de las actividades productivas, en el largo plazo, será el único medio del que dispondrán las autoridades competentes para prevenir que los agricultores de las parroquias no destruyan los únicos recursos que les quedan para poder subsistir, producto de la inseguridad alimentaria y falta de apoyo gubernamental.

Con la finalidad de identificar las expectativas de los moradores locales con respecto al mejoramiento de sus condiciones de vida y promover la sustentabilidad a largo plazo de las acciones iniciadas para la conservación de la especie Cordiaalliodora, se realizaron varias reuniones de consulta con grupos seleccionados y un taller de trabajo.

Una ordenación de restablecimiento forestal y recuperación de las fincas cafetaleras, mediante la ejecución de sistemas agroforestales con especies de uso múltiple y la práctica de técnicas de producción amigables con el ambiente, otorgaría incentivos a los pobladores de las parroquias para sustituir las actuales prácticas tradicionales de uso de la finca por buenas prácticas de manejo, que hagan factibles la producción con la preservación.

\section{CONCLUSIONES}


- La percepción de los pobladores y responsables del manejo, sobre las funciones y aportes de los bosques secundarios en los agroecosistemas cafetaleros del Cantón Jipijapa, es baja.

- El diseño de la estrategia para la gestión sostenible de los bosques ocupados por fincas agroforestales del cantón Jipijapa tiene un carácter participativo y define los lineamientos fundamentales para mitigar los impactos ambientales derivados de prácticas inadecuadas de manejo.

\section{BIBLIOGRAFÍA}

Alegre, Ada. Gestión y Evaluación de Impacto Ambiental en Proyectos de Inversión.

La Evaluación de Impacto Ambiental en la Gestión Ambiental Peruana. Cursoadaptado al Programa de capacitación BID, 2001. 2002.

Amezaga, I. and Onaindia, M. The effect of energreen and deciduous coniferous plantations end de fielloyer and seed bank of native woodlands. Ecogrphy 20, 308 318. 1997.

Arcia, M. y Garibaldi, C. Los Bosques, Bienes y Servicios Ambientales de la Reserva Forestal El Montuoso, provincia de Herrera, Panamá. En C. Garibaldi (Ed.), Diversidad Biológica y Servicios Ambientales de los Fragmentos de Bosques en la Reserva Forestal El Montuoso, Panamá, pp 173-193. 2005.

Espinoza, N., Gatica, J. y Smyle, J.El pago de servicios ambientales y el desarrollo sostenible en el medio rural. (Documento de Trabajo). San José, Costa Rica: RUTA, IICA, Serie de publicaciones. 1999.

FAO a. Contribución de los Bosques a los Objetivos de Desarrollo del Milenio /17 Periodo de Sesiones). Roma, Italia: Comité de Montes. FAO 2005

FAO b. Situación de los Bosques del Mundo. Roma, Italia. FAO 2005. 
García, C. Establecimiento de Plantaciones Forestales. Eucalyptus sp. INFOR-FDI. 32 pp. 2006.

Holl KL, M Lin \& E Samuels. 2000. Tropical montaneforestrestorationinCostaRica:overcoming barriers to dispersalandestablishment.Restoration Ecology8: 339-349.

Guariguata, M. R. y Kattan, G. H. Ecología y Conservación de Bosques Neotropocales LUR. Costa Rica 691.pp. 2002.

Grijalva, J.; Checa, X.; Ramos, R.; Barrera, P.; Limongi, R. 2012. Situación de los Recursos Genéticos Forestales - Informe País Ecuador. Preparado por el Programa Nacional de Forestería del INIAP con aval del INIAP/FAO/MAE/MAGAP/MMRREE. Documento sometido a la Comisión Forestal de la FAO-Roma, para preparación del Primer Informe sobre el Estado de los Recursos Genéticos Forestales en el Mundo. p 95.

Laurance, W. F., y Bierregaard, M. A. Tropical Forest Remnants: Ecology, Conservation andManagement of Fragmented Communities. Univ. of Chicago Press, Chicago. 1997.

Ministerio de Agricultura y Ganadería 2003 Censo agropecuario en la Provincia de Manabí.

Mc Neely, J. A. Economics and Biological Diversity: Developing and Using Economic Incentives to Conserve Biological Resources. IUCN. Gland, Switzerland, xiv+232 pp. 1988.

OIMT. Organización Internacional de las Maderas Tropicales. Estado de Ordenación de los Bosques Tropicales. ITTO. Technical Series No. 24, 2. 2002. 
Quédraogo, M. Estrategia de Conservación Intraespecífica para PinuscaribaeaMoreletvar. CaribaeaBarret y Golfari. 1997.

Scherr, J. A. New Agenda for Forest Conservation and Poverty Alleviation: Making Markerts Work for Low-income Producers. Washington, D.C: Forest Trends. 2003 